\title{
Microscopic and phenomenological models of driven systems in structured reservoirs
}

\author{
Gian Luca Giorgi $\odot,{ }^{1,2}$ Astghik Saharyan $\odot,{ }^{3}$ Stéphane Guérin $\odot,{ }^{3}$ Dominique Sugny $\odot,{ }^{3}$ and Bruno Bellomo $\oplus^{1, *}$ \\ ${ }^{1}$ Institut UTINAM, CNRS UMR 6213, Université Bourgogne Franche-Comté, Observatoire des Sciences de l'Univers THETA, 41 bis avenue \\ de l'Observatoire, F-25010 Besançon, France \\ ${ }^{2}$ IFISC (UIB-CSIC), Instituto de Física Interdisciplinar y Sistemas Complejos, UIB Campus, E-07122, Palma de Mallorca, Spain \\ ${ }^{3}$ Laboratoire Interdisciplinaire Carnot de Bourgogne, CNRS UMR 6303, Université Bourgogne Franche-Comté, \\ BP 47870, F-21078 Dijon, France
}

(Received 8 August 2019; published 24 January 2020)

\begin{abstract}
We study the paradigmatic model of a qubit interacting with a structured environment and driven by an external field by means of a microscopic and a phenomenological model. The validity of the so-called fixed-dissipator (FD) assumption, where the dissipation is taken as the one of the undriven qubit, is discussed. In the limit of a flat spectrum, the FD model and the microscopic one remarkably practically coincide. For a structured reservoir, we show in the secular limit that steady states can be different from those determined from the FD model, opening the possibility for exploiting reservoir engineering. We explore it as a function of the control field parameters, of the characteristics of the spectral density, and of the environment temperature. The observed widening of the family of target states by reservoir engineering suggests new possibilities in quantum control protocols.
\end{abstract}

DOI: 10.1103/PhysRevA.101.012122

\section{INTRODUCTION}

Generally speaking, no quantum system can be considered as completely isolated from its environment, which is at the origin of dissipation and decoherence [1,2]. These dissipative processes could negatively influence control protocols which aim at bringing a quantum system toward a desired target state, such as the ones considered in quantum control [3-9] and in remote state preparation [10,11]. Dissipative dynamics can be strongly modified by using, for instance, dynamical decoupling strategies [12-14] or tuned into a useful tool, e.g., by properly engineering the characteristics of the environment, to generate specific states [15-18].

The study of open quantum systems usually involves approximations [1]. Master equations are often derived in the weak-coupling regime between the system and the bath (Born approximation) and for memoryless dynamics with time-independent dissipation rates (Markovian approximation). Other common assumptions concern the absence of initial correlations between the system and its environment and the secular approximation. A standard way to obtain a master equation is based on a microscopic approach which takes into account the full Hamiltonian of the system and the environment, including their mutual coupling, and by performing all or some of the approximations described above. The system dynamics is completely positive as long as the master equation is in the Lindblad form [19,20].

An alternative route to take into account environmental effects relies on a phenomenological description of standard dissipative and dephasing mechanisms where Lindblad superoperators are designed to reproduce the desired process and the dissipator is built "by hand," for instance, by inferring the

\footnotetext{
*bruno.bellomo@univ-fcomte.fr
}

decay rates from experimental data. This approach may lead to a drastic simplification of the dynamics [1]. However, there are scenarios for which the phenomenological technique may not be reliable, as it may not be able to capture all the relevant aspects of the dynamics. In particular, this problem becomes crucial when an external control field is exerted to the system or when different systems are coupled to each other while dissipating locally [21-23].

In the context of phenomenological modeling of open quantum systems subject to external control fields, a standard assumption is the so-called fixed-dissipator (FD) assumption [24-28]. This is based on the hypothesis that the dissipative part of the master equation is not changed by the control term. Comparisons between phenomenological and microscopic master equations have been realized, also considering, in the case of bipartite systems, the effects due to a strong coupling between the internal parts [29-33]. Comparisons have been done also in the context of adiabatic population transfer in three-level systems, pointing out some relevant differences [34-36]. Problematic consequences of phenomenological master equations in quantum thermodynamics have been recently discussed [22,37,38] and, as shown in Ref. [39], the FD assumption can be at the origin of nonphysical trajectories in the non-Markovian limit. A generalized approach trying to unify phenomenological and microscopic approaches has been recently proposed [40].

The scope of this paper is to study the case of a driven qubit interacting with a structured environment by means of a microscopic model and to analyze the consequences of the FD assumption. This includes the possibility of using reservoir engineering as a tool for quantum control. For that purpose, we mainly study the dynamics on asymptotic timescales and compare the steady states reachable with a microscopic master equation (MME) with the ones given by a master equation based on a fixed dissipator (FDME). We show that 
manipulating the environment through reservoir engineering, which is possible when the environment spectrum is not flat, allows one to obtain a collection of stationary states that can be very different from the ones given by the FDME.

The paper is organized as follows. In Sec. II, we introduce the model of a qubit driven by a monochromatic laser and interacting with a bosonic environment. In Sec. III, the MME for such a system is explicitly derived, with some technical details reported in the Appendix. In Sec. IV, we review the FD assumption, while in Sec. V, we present the main results of this study in the case of structured environments, both at zero and nonzero temperatures. Conclusive remarks and prospective views are presented in Sec. VI.

\section{THE MODEL SYSTEM}

For the sake of simplicity, we tackle the problem of comparing microscopic and phenomenological models of driven systems in structured environments by revisiting a simple quantum system made of a qubit of frequency $\omega_{0}$ driven by a monochromatic control laser field whose frequency is $\omega_{L}$ and whose initial phase is $\varphi[41,42]$. We define the detuning as $\Delta=\omega_{0}-\omega_{L}$ and we refer to the Rabi frequency $\Omega$, related to the intensity of the laser field, as the driving amplitude. We assume henceforth that $\omega_{0}$ and $\omega_{L}$ are much larger than $\Delta$ and $\Omega$. The starting Hamiltonian is given by

$$
\bar{H}_{S}=\frac{\hbar \omega_{0}}{2} \sigma_{z}+\hbar \Omega \cos \left(\omega_{L} t+\varphi\right) \sigma_{x},
$$

where $\sigma_{z}$ and $\sigma_{x}$ are Pauli matrices. Under the above condition on the parameters we may apply the rotating wave approximation on $\bar{H}_{S}$, obtaining

$$
\bar{H}_{S}^{R W}=\frac{\hbar \omega_{0}}{2} \sigma_{z}+\frac{\hbar \Omega}{2}\left[e^{-i\left(\omega_{L} t+\varphi\right)} \sigma_{+}+e^{+i\left(\omega_{L} t+\varphi\right)} \sigma_{-}\right],
$$

where $\sigma_{+}$and $\sigma_{-}$are, respectively, the raising and the lowering qubit operator. We also move to a frame rotating at frequency $\omega_{L}$, by means of the unitary operator $U_{L}=$ $\exp \left[-i\left(\omega_{L} t+\varphi\right) \sigma_{z} / 2\right]$ (also absorbing the time-independent phase factor $\varphi$ ). In such a rotating frame, any state $|\bar{\psi}\rangle$ is mapped into $|\psi\rangle=U_{L}^{\dagger}|\bar{\psi}\rangle$, and the Schrödinger equation $i \hbar \partial_{t}|\bar{\psi}\rangle=\bar{H}_{S}^{R W}|\bar{\psi}\rangle$ becomes $i \hbar \partial_{t}\left(U_{L}|\psi\rangle\right)=\bar{H}_{S}^{R W} U_{L}|\psi\rangle$, that can be rewritten as $i \hbar \partial_{t}|\psi\rangle=H_{S}|\psi\rangle$, where $H_{S}=$ $U_{L}^{\dagger} \bar{H}_{S}^{R W} U_{L}-i \hbar\left(\partial_{t} U_{L}^{\dagger}\right) U_{L}$, which leads to

$$
H_{S}=\frac{\hbar \Delta}{2} \sigma_{z}+\frac{\hbar \Omega}{2} \sigma_{x} .
$$

The interaction between the system and the environment, which is assumed not to depend on the control field (see, for instance, Ref. [43]), reads as follows:

$$
\bar{H}_{I}=\sum_{k} \hbar\left(g_{k} a_{k}+g_{k}^{*} a_{k}^{\dagger}\right) \sigma_{x},
$$

where $a_{k}$ and $a_{k}^{\dagger}$ are, respectively, the annihilation and the creation operators of the bosonic bath and $g_{k}$ are the coupling constants. In the above rotating frame, $\bar{H}_{I}$ becomes $H_{I}=$ $U_{L}^{\dagger} \bar{H}_{I} U_{L}$, which gives

$$
H_{I}=\sum_{k} \hbar\left(g_{k} a_{k}+g_{k}^{*} a_{k}^{\dagger}\right)\left[e^{i\left(\omega_{L} t+\varphi\right)} \sigma_{+}+e^{-i\left(\omega_{L} t+\varphi\right)} \sigma_{-}\right] .
$$

The free Hamiltonian of the environment has the form $H_{E}=$ $\sum_{k} \hbar \omega_{k} a_{k}^{\dagger} a_{k}$ and, of course, is not touched by the change of frame. $H_{S}$ can be diagonalized as $H_{S}=\frac{\hbar v}{2}\left(\left|\phi_{+}\right\rangle\left\langle\phi_{+}\right|-\right.$ $\left.\left|\phi_{-}\right\rangle\left\langle\phi_{-}\right|\right)$, with $v=\sqrt{\Delta^{2}+\Omega^{2}}$. Its eigenstates are

$$
\left|\phi_{+}\right\rangle=C|e\rangle+S|g\rangle, \quad\left|\phi_{-}\right\rangle=C|g\rangle-S|e\rangle,
$$

where $|g\rangle$ and $|e\rangle$ are, respectively, the ground and the excited states of the qubit free Hamiltonian $\left(\hbar \omega_{0} / 2\right) \sigma_{z}, C=\cos (\theta / 2)$, $S=\sin (\theta / 2)$, and

$$
\theta=2 \arctan [(v-\Delta) / \Omega] .
$$

For example, for a given $\Delta>0, \theta$ goes from 0 to $\pi / 2$ when $\Omega$ goes from 0 to infinity.

\section{MICROSCOPIC MASTER EQUATION}

To derive a microscopic master equation, the qubit driven by the field is treated first. The resulting dressed qubit is next coupled to the environment by expressing $H_{I}$ in terms of the eigenoperators of $H_{S}$ and the standard Born and Markov approximations are applied (see also Refs. [41,42]).

Defining $\tilde{\sigma}_{z}=\left|\phi_{+}\right\rangle\left\langle\phi_{+}|-| \phi_{-}\right\rangle\left\langle\phi_{-}\right|$and $\tilde{\sigma}_{ \pm}=\left|\phi_{ \pm}\right\rangle\left\langle\phi_{\mp}\right|$, these are connected to $\sigma_{z}, \sigma_{+}$, and $\sigma_{-}$by

$$
\sigma_{ \pm}=C^{2} \tilde{\sigma}_{ \pm}-S^{2} \tilde{\sigma}_{\mp}+S C \tilde{\sigma}_{z}, \quad \sigma_{z}=\cos \theta \tilde{\sigma}_{z}-\sin \theta \tilde{\sigma}_{x} .
$$

Using the expressions for $\sigma_{+}$and $\sigma_{-}$, the qubit operators in $H_{I}$ can be written in terms of eigenoperators of $H_{S}$. The detailed derivation of the MME is presented in the Appendix. Its final form in the Schrödinger picture is

$$
\dot{\rho}=-\frac{i}{\hbar}\left[H_{S}+H_{L S}, \rho\right]+\mathcal{D}^{\mathrm{sec}}(\rho)+\mathcal{D}^{\mathrm{nsec}}(\rho),
$$

where $H_{L S}$ is the Lamb shift Hamiltonian, whose role is discussed in the Appendix, while $\mathcal{D}^{\sec }(\rho)$ and $\mathcal{D}^{\text {nsec }}(\rho)$ are, respectively, the secular and the nonsecular parts of the dissipator, the latter featuring terms oscillating at frequencies $v$ and $2 v$.

With regards to $\mathcal{D}^{\sec }(\rho)$, it is given by

$$
\mathcal{D}^{\mathrm{sec}}(\rho)=\gamma_{-}^{\theta} \mathcal{L}\left[\tilde{\sigma}_{+}\right](\rho)+\gamma_{+}^{\theta} \mathcal{L}\left[\tilde{\sigma}_{-}\right](\rho)+\gamma_{z}^{\theta} \mathcal{L}\left[\tilde{\sigma}_{z}\right](\rho),
$$

where the Lindblad superoperator is $\mathcal{L}[\hat{X}](\rho)=\hat{X} \rho \hat{X}^{\dagger}-$ $\left\{\rho, \hat{X}^{\dagger} \hat{X}\right\} / 2$, with

$$
\begin{aligned}
\gamma_{-}^{\theta}= & 2 \pi\left\{C^{4} J\left(\omega_{L}+v\right) n\left(\omega_{L}+v\right)+S^{4} J\left(\omega_{L}-v\right)\right. \\
& \left.\times\left[1+n\left(\omega_{L}-v\right)\right]\right\} \\
\gamma_{+}^{\theta}= & 2 \pi\left\{C^{4} J\left(\omega_{L}+v\right)\left[1+n\left(\omega_{L}+v\right)\right]+S^{4} J\left(\omega_{L}-v\right)\right. \\
& \left.\times n\left(\omega_{L}-v\right)\right\} \\
\gamma_{z}^{\theta}= & 2 \pi\left\{S^{2} C^{2} J\left(\omega_{L}\right)\left[1+2 n\left(\omega_{L}\right)\right]\right\}
\end{aligned}
$$

where $J(\omega)$ is the spectral density of the environment and $n(\omega)=1 /\left[e^{\hbar \omega /\left(k_{B} T\right)}-1\right]$ is the average number of excitations in the bath at frequency $\omega$, with $k_{B}$ being the Boltzmann constant. The above coefficients can be rewritten as

$$
\begin{aligned}
& \gamma_{-}^{\theta}=C^{4} \gamma_{+} n_{+}+S^{4} \gamma_{-}\left(1+n_{-}\right), \\
& \gamma_{+}^{\theta}=C^{4} \gamma_{+}\left(1+n_{+}\right)+S^{4} \gamma_{-} n_{-}, \\
& \gamma_{z}^{\theta}=S^{2} C^{2} \gamma_{0}\left(1+2 n_{0}\right),
\end{aligned}
$$


where $\gamma_{p}=2 \pi J\left(\omega_{L}+p v\right)$ and $n_{p}=n\left(\omega_{L}+p v\right)$, where $p=$ $\{-1,+1,0\}$ (for any parameter $l$ depending on $p$ we use the shorthand notation $l_{-1}=l_{-}$and $l_{+1}=l_{+}$). Because of the control field, the qubit experiences dephasing- and thermal-like dissipative effects already in the case of a zerotemperature bath $\left(n_{p}=0\right)$, as shown by the fact that also in this case all the $\gamma_{p}^{\theta}$ contribute to the dynamics and the steady state is not expected to be pure anymore.

The operator $\mathcal{D}^{\text {nsec }}(\rho)$ and its coefficients are reported in the Appendix. In Sec. VA, we give some comments about when their effect cannot be neglected. A detailed analysis of the limits of validity of the secular approximation in our system can be found in Ref. [44].

\section{THE REFERENCE CASE: THE FIXED DISSIPATOR}

In Sec. III, we have seen that, in the microscopic approach, the dissipator depends on the control field acting on the qubit. The FD approach consists in neglecting this dependance and in assuming that the dissipative part of the master equation is equal to the one in the absence of the control field, i.e., the qubit coupled to the environment is treated first, and this single entity is next coupled to the laser. The application of this procedure is well known in quantum control protocols (see, e.g., Refs. [24-28]). Recently, this approach has been used to determine the control Hamiltonian that counteracts a given dissipation $[25,28]$. In this context, we consider a density matrix evolving according to a general Lindblad master equation

$$
\dot{\rho}=-\frac{i}{\hbar}[H, \rho]+\mathcal{D}^{\mathrm{fd}}(\rho) .
$$

The set of stationary solutions $\dot{\rho}^{\text {fd }}=0$, which are compatible with the fixed dissipator $\mathcal{D}^{\mathrm{fd}}\left(\rho^{\mathrm{fd}}\right)$ can be computed by disregarding the coherent part. Since the coherent part of the master equation cannot change the spectrum and then the purity of the state, the same must also be true for the dissipator $[25,26,28]$. Then, the collection of stationary states $\rho^{\mathrm{fd}}$ must obey the relation

$$
\operatorname{Tr}\left\{\left(\rho^{\mathrm{fd}}\right)^{n-1} \mathcal{D}^{\mathrm{fd}}\left(\rho^{\mathrm{fd}}\right)\right\}=0, \quad \forall n \in\{2, \ldots, d\},
$$

where $d$ is the dimension of the Hilbert space. Thus, we have defined a fixed dissipator and a family of Hamiltonians. For any steady state, we can find the Hamiltonian $H$ such that $\dot{\rho}^{\mathrm{fd}}=0$. Writing the steady state as $\rho^{\mathrm{fd}}=\sum_{\alpha=1}^{d} \lambda_{\alpha}|\alpha\rangle\langle\alpha|$, it follows that $[25,28]$

$$
H=\hbar \sum_{\alpha, \beta: \lambda_{\alpha} \neq \lambda_{\beta}} \frac{i\left\langle\alpha\left|D^{\mathrm{fd}}\left(\rho^{\mathrm{fd}}\right)\right| \beta\right\rangle}{\lambda_{\alpha}-\lambda_{\beta}}|\alpha\rangle\langle\beta| .
$$

For the case of the qubit introduced in Sec. II, we only need to satisfy $\operatorname{Tr}\left\{\rho^{\mathrm{fd}} \mathcal{D}^{\mathrm{fd}}\left(\rho^{\mathrm{fd}}\right)\right\}=0$. In this model, at a given bath temperature $T$, the FD is equal to the dissipator one would obtain in the absence of the control field $(\Omega \rightarrow 0)$. This can be obtained from the microscopic dissipator of Eq. (9) taking $\theta=0$ and $v=\Delta$. In this limit, the decay rates of Eq. (12) tend to $\gamma_{-}^{0}=\gamma_{\mathrm{fd}} n_{\mathrm{fd}}, \gamma_{+}^{0}=\gamma_{\mathrm{fd}}\left(1+n_{\mathrm{fd}}\right)$, and $\gamma_{z}^{0}=0$, where $\gamma_{\mathrm{fd}}=$ $2 \pi J\left(\omega_{0}\right)$ and $n_{\mathrm{fd}}=n\left(\omega_{0}\right)$. The fixed dissipator is then of the

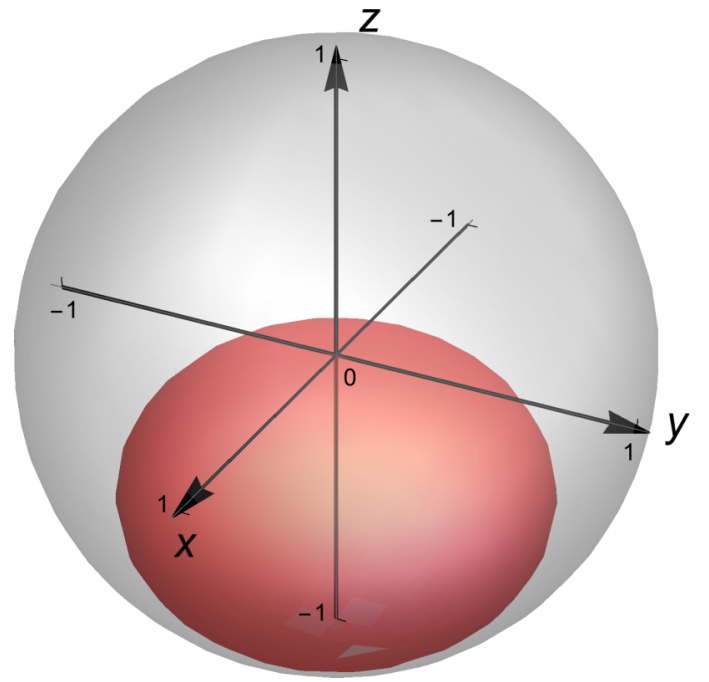

FIG. 1. Bloch sphere (gray) and steady-state ellipsoid (red). The components of the Bloch vector of an arbitrary state $\rho$ are $r_{x}=$ $2 \operatorname{Re}\left[\rho_{e g}\right], r_{y}=-2 \operatorname{Im}\left[\rho_{e g}\right]$, and $r_{z}=2 \rho_{e e}-1$. The ellipsoid has been drawn by taking $T=0$.

form

$$
D^{\mathrm{fd}}(\rho)=\gamma_{\mathrm{fd}} n_{\mathrm{fd}} \mathcal{L}\left[\sigma_{+}\right](\rho)+\gamma_{\mathrm{fd}}\left(1+n_{\mathrm{fd}}\right) \mathcal{L}\left[\sigma_{-}\right](\rho) .
$$

It follows that in the FD approach the steady state depends on $\gamma_{\mathrm{fd}}$. To compute it, we use $H=H_{S}$ in Eq. (13), neglecting the Lamb shift. We observe that when it is present and not negligible, it could be taken into account by considering that it just leads to a different value of the parameter $\Delta$. In this sense, in all the parts where the result depends only on the ratio $\Omega / \Delta$, the same result could be obtained by just changing $\Omega$ according to the change of $\Delta$. In particular, in the limit of flat spectrum, it is possible to see that the Lamb shift computed in this phenomenological framework is zero (see the Appendix for an explanation of how this can be found in the microscopic approach). The nonsecular terms that one could obtain using a microscopic derivation applied to the qubit coupled to the environment in the absence of the driving laser will not be considered here since the FDME is introduced on phenomenological grounds. The steady states can be then expressed in the $\sigma_{z}$ basis in terms of some of its density matrix elements (hereafter we use the notation $\rho_{i j}=\langle i|\rho| j\rangle$ ) as (restoring the dependence on $\varphi$ )

$$
\begin{aligned}
& \rho_{e e}^{\mathrm{fd}}=\frac{n_{\mathrm{fd}}}{1+2 n_{\mathrm{fd}}}+\frac{\Omega^{2} /\left(1+2 n_{\mathrm{fd}}\right)}{\gamma_{\mathrm{fd}}^{2}\left(1+2 n_{\mathrm{fd}}\right)^{2}+4 \Delta^{2}+2 \Omega^{2}}, \\
& \rho_{e g}^{\mathrm{fd}}=-\Omega \frac{2 \Delta /\left(1+2 n_{\mathrm{fd}}\right)+i \gamma_{\mathrm{fd}}}{\gamma_{\mathrm{fd}}^{2}\left(1+2 n_{\mathrm{fd}}\right)^{2}+4 \Delta^{2}+2 \Omega^{2}} e^{-i \varphi},
\end{aligned}
$$

being, of course, for any $\rho, \rho_{g g}=1-\rho_{e e}$ and $\rho_{g e}=\rho_{e g}^{*}$. The FD steady solutions by varying the control field parameters, $\Omega, \Delta$, and $\varphi$, are represented in Fig. 1 (for $T=0$ ) where they are shown to lie on the surface of an ellipsoid inside the Bloch sphere $[25,26,28,45]$. This ellipsoid is a standard geometric structure in nuclear magnetic resonance $[26,46,47]$. For $T \neq$ 0 , the steady states lie on a smaller ellipsoid inside the one depicted in Fig. 1. 


\section{RESERVOIR ENGINEERING THROUGH MICROSCOPIC MASTER EQUATION WITH STRUCTURED ENVIRONMENT}

We present in this section the control of steady states by using the MME of Sec. III. In particular, we compare the steady-state solutions of the FDME with the ones provided by the MME to discuss how the control of the system is modified when the environment is used as a tool to suitably tailor the asymptotic states. We also compare some specific dynamics to highlight our results.

In the case of flat spectrum, it holds $\gamma_{-}=\gamma_{+}=\gamma_{0}=$ $\gamma_{\mathrm{fd}}$ and one can show the remarkable property that, under the approximation $n_{-} \approx n_{+} \approx n_{0} \approx n_{\mathrm{fd}}$, the MME coincides exactly with the FDME [41] (see the Appendix for a complete derivation). The steady states of the MME and of the FDME are thus the same for any $T$. In particular, in the secular limit, in the frame rotating at the laser frequency (after restoring the phase $\varphi$ ), the MME steady solutions are equal to the ones of Eq. (17) after discarding the terms containing $\gamma_{\mathrm{fd}}$, which are indeed negligible in this limit. One can show that the geometric form of the steady-state solutions obtained by varying the control field parameters, $\Omega, \Delta$, and $\varphi$, corresponds to the very same ellipsoid of Fig. 1 . When nonsecular terms are added, the microscopic steady states coincide with the ones obtained with the FD, given in Eq. (17). We consider below the case of structured environments in which relevant differences can instead occur.

We consider in particular the MME in the secular regime, noting that this regime is typically encountered in several contexts such as in quantum optics setups [1]. The steady state $\rho^{\mathrm{sec}}$, which satisfies both $\left[\rho^{\mathrm{sec}}, H_{S}+H_{L S}\right]=0$ and $\mathcal{D}^{\sec }\left(\rho^{\mathrm{sec}}\right)=0$, is

$$
\rho^{\mathrm{sec}}=\frac{\gamma_{-}^{\theta}}{\gamma_{+}^{\theta}+\gamma_{-}^{\theta}}\left|\phi_{+}\right\rangle\left\langle\phi_{+}\left|+\frac{\gamma_{+}^{\theta}}{\gamma_{+}^{\theta}+\gamma_{-}^{\theta}}\right| \phi_{-}\right\rangle\left\langle\phi_{-}\right|,
$$

where the superscript "sec" refers to the secular master equation. The collection of steady states that are obtained as functions of the control parameter $\theta$ and of the phase $\varphi$ (once it is restored) describes a surface in the Bloch vector representation which is invariant under a rotation around the $z$ axis.

We consider structured environments characterized by a spectral density varying notably around $\omega_{L}$ on the scale of the dressed frequency $v$. In this scenario, even in the limit where the secular approximation holds, the microscopic approach provides target steady states that may be not close to the ones obtained with the FDME. In the Markovian limit, while in the FD case there is only one value of the spectral density that matters, the two additional sidebands at $\omega_{L} \pm v$ must be considered according to the microscopic derivation [see Eq. (11)].

When $n_{-} \approx n_{+} \approx n_{0} \approx n_{\mathrm{fd}}$, Eq. (18) gives (after restoring the phase $\varphi$ )

$$
\begin{aligned}
& \rho_{e e}^{\mathrm{sec}} \approx \frac{n_{\mathrm{fd}}}{1+2 n_{\mathrm{fd}}}+\frac{S^{2} C^{2}}{1+2 n_{\mathrm{fd}}} \frac{S^{2} \gamma_{-}+C^{2} \gamma_{+}}{S^{4} \gamma_{-}+C^{4} \gamma_{+}}, \\
& \rho_{e g}^{\mathrm{sec}} \approx \frac{S C}{1+2 n_{\mathrm{fd}}} \frac{S^{4} \gamma_{-}-C^{4} \gamma_{+}}{S^{4} \gamma_{-}+C^{4} \gamma_{+}} e^{-i \varphi} .
\end{aligned}
$$

Exploiting the dependence on the two frequencies $\omega_{L} \pm v$ opens the possibility for taking profit from reservoir engineering. It indeed allows one to deform the ellipsoid of Fig. 1, thus modifying the family of target states. For instance, one of the possible consequences is that the equator of the ellipsoid can be broadened, allowing one to get higher values for the absolute value of the nondiagonal elements of the density matrix, as it is always possible to reduce the weight of the smaller term in Eq. (18) and then to obtain purer states. We observe that $2\left|\rho_{e g}\right|$ is a measure of the resource named quantum coherence [48].

\section{A. The case of zero temperature}

We start the analysis with the zero-temperature case. The scenarios where $J\left(\omega_{L}+v\right) \gtrless J\left(\omega_{L}-v\right)$ are compared with the flat spectral density case in Fig. 2, where the components $x$ and $z$ of the Bloch vector of the steady states, $r_{x}=2 \operatorname{Re}\left[\rho_{e g}\right]$ and $r_{z}=2 \rho_{e e}-1$, are plotted. In Fig. 2(a), we consider fixed values for the ratio $\mu=\gamma_{-} / \gamma_{+}=J\left(\omega_{L}-v\right) / J\left(\omega_{L}+v\right)$ for all values of the dressed energy. This analysis permits to visualize for any value of the control parameters $\theta$ of Eq. (7) (or equivalently of $\Omega / \Delta$ ) and $\varphi$ how much the steady states differ in the two approaches for a given $\mu$. In the two panels, all the parts of the different lines are obtained by considering a fixed positive $\Delta$ and using $\Omega$, assuming values $\geqslant 0$, and $\varphi$, being equal to 0 or $\pi$, as control parameters. In particular, with respect to the FDME, purer states can be obtained $\left(\left|\rho_{e g}\right|\right.$ may become closer to the maximum allowed value of $1 / 2$ ) and even the population inversion can be reached. We observe that the FDME is considered in the case when its dependence on $\gamma_{\mathrm{fd}}$ is negligible, and so the steady state practically coincides with the microscopic secular one in the limit of flat spectrum (or more in general when $\mu=1$ ).

As an example, let us consider the case where the target state reached using the FD dynamics at zero temperature is one of the states with maximally allowed $\left|\rho_{e g}\right|$, that is, a point that lies on its equator [25,28]. This class of states is obtained using $\Omega= \pm \sqrt{2} \Delta$ and, written in the Bloch form, is $\vec{r}_{\mathrm{mc}}=\{\mp \cos \varphi / \sqrt{2}, \mp \sin \varphi / \sqrt{2},-1 / 2\}$. We focus on the case $\Omega=\sqrt{2} \Delta$ and $\varphi=\pi$, obtaining then $\vec{r}_{\mathrm{mc}}=$ $\{1 / \sqrt{2}, 0,-1 / 2\}$. On the other hand, in the presence of structured reservoir, taking $\Omega=\sqrt{2} \Delta$ and $\varphi=\pi$, we would end up in $\vec{r} \simeq\{0.805,0,-0.569\}$ using $\mu=0.1$ or in $\vec{r} \simeq$ $\{0.134,0,-0.095\}$ using $\mu=10$. The three states, reached with the same control field, are visualized with points in Fig. 2(a). The distances between these points clearly point out how much could be the error due to using the FDME to predict the steady state in a given control protocol.

In order to treat a specific physical scenario where $\mu$ varies when the control field parameters are changed, we now consider the case in which the spectral density has the Lorentzian profile

$$
J_{\text {Lor }}(\omega)=\frac{\gamma_{l}}{2 \pi} \frac{\lambda^{2}}{\left(\omega-\omega_{c}\right)^{2}+\lambda^{2}},
$$

where the parameter $\lambda$ defines the width of the curve and $\omega_{c}$ its center. We consider values of $\lambda$ much greater than $\gamma_{l}$, such that the Markovian approximation used for the derivation of the MME is satisfied. The flat spectral density case is recovered 

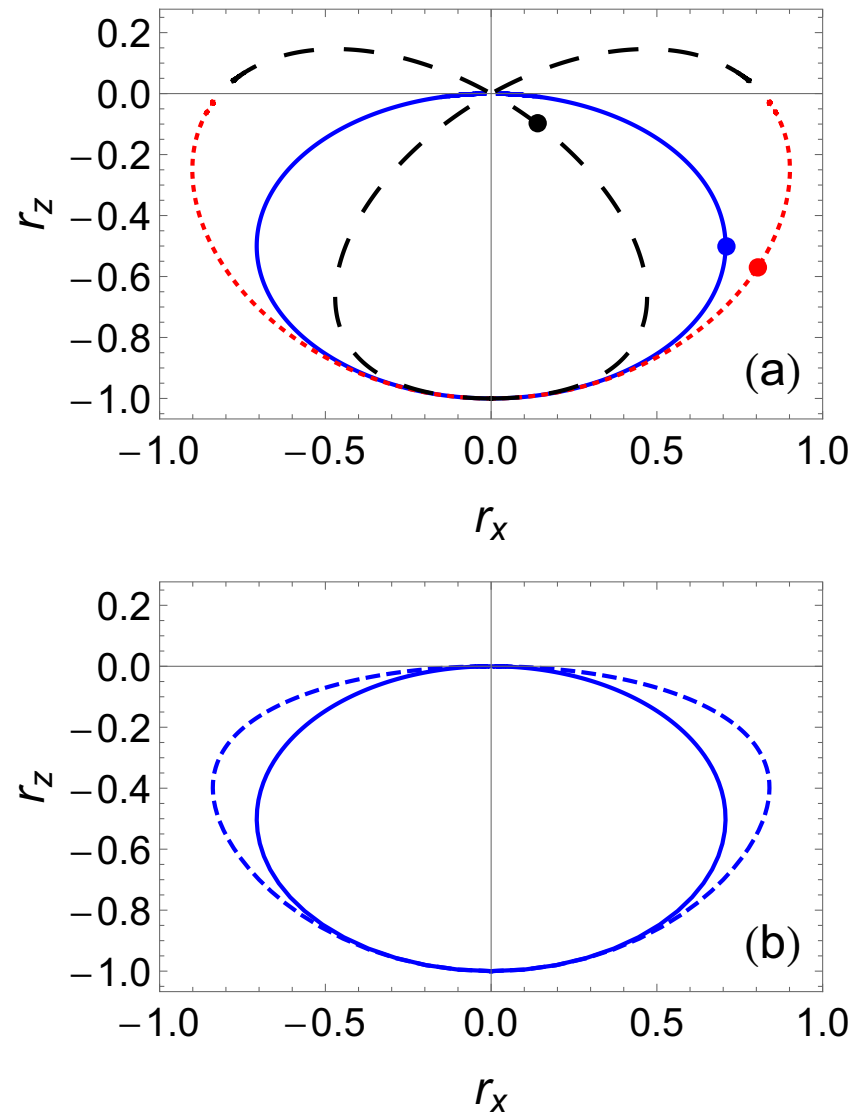

FIG. 2. Families of steady states (components $x$ and $z$ of the Bloch vector, $r_{x}$ and $r_{z}$ ) determined from the FDME (the dependence on $\gamma_{\mathrm{fd}}$ is assumed to be negligible) and from the secular MME by varying the control field parameters $\Omega$, assuming values $\geqslant 0$, and $\varphi$, being equal to 0 or $\pi$, for a positive fixed value of $\Delta$. (a) The FDME case is represented by the blue solid line while the red dotted and the black dashed lines represent the microscopic steady states when $\mu=\gamma_{-} / \gamma_{+}$is kept fixed for any $v$ and equal to, respectively, 0.1 and 10 . The three enlightened points represent the three steady states obtained by using $\Omega=\sqrt{2} \Delta$ and $\varphi=\pi$, which, in the FDME case, gives the maximum allowed $\left|\rho_{e g}\right|$. (b) The family of stationary states has been calculated either assuming the FDME (solid line) or the Lorentzian density of states given in Eq. (20) with $\omega_{c}=\omega_{0}$ and $\lambda=\Delta$ (dashed line).

in the limit $\lambda \rightarrow \infty$. Using Eq. (20), one can expect that only in some parts of the parameter space the deformation is relevant. On the tails of the curve, we fall for instance in something similar to the flat spectral density case, which gives the same results of the FDME. The differences in the case of a Lorentzian spectral density are depicted in Fig. 2(b), where we have assumed that the dependence on $\gamma_{\mathrm{fd}}$ of the FDME steady solutions is negligible and calculated the steady states by varying $\Omega / \Delta$ in the case of a fixed Lorentzian, with $\lambda=\Delta$ (we have fixed a positive value for $\Delta$ and varied $\Omega$, assuming values $\geqslant 0$, and $\varphi$, being equal to 0 or $\pi$ ).

We have numerically compared the secular MME curve in Fig. 2(b) with the one obtained by adding the nonsecular terms at zero temperature and for values of $\gamma_{\mathrm{fd}}$ much smaller than $\Delta$. In general, the nonsecular curve is very close to the MME curve except when one approaches the origin of the

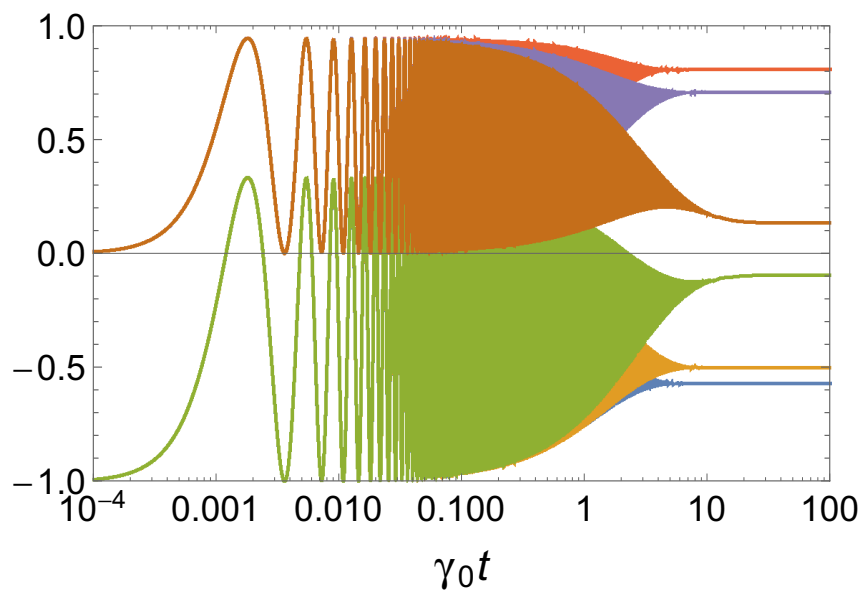

FIG. 3. The Bloch vector components $r_{x}$ and $r_{z}$, as a function of time (in units of $\gamma_{0}^{-1}$ and in logarithmic scale). The initial state is $|g\rangle$, $\Omega=\sqrt{2} \Delta, \varphi=\pi$, and $\gamma_{0}=0.001 \Delta$. Three cases are considered: the MME with $\mu=0.1\left(\gamma_{-}=0.2 \gamma_{0}\right.$ and $\left.\gamma_{+}=2 \gamma_{0}\right)$, the FDME with $\gamma_{\mathrm{fd}}=\gamma_{0}$, and the MME with $\mu=10\left(\gamma_{-}=2 \gamma_{0}\right.$ and $\left.\gamma_{+}=0.2 \gamma_{0}\right)$. All the $r_{x}$ curves start from 0 and reach the larger value for the case $\mu=0.1$, the intermediate one for the FDME, and the lower one for the case $\mu=10$. All the $r_{z}$ curves start from -1 and reach the larger value (in modulus) for the case $\mu=0.1$, the intermediate one (in modulus) for the FDME, and the lower one (in modulus) for the case $\mu=10$. The fixed dissipator dynamics is practically identical to the one of the MME in the limit of flat spectrum, $\gamma_{-}=\gamma_{+}=\gamma_{0}=\gamma_{\mathrm{fd}}$ $(\mu=1)$.

axis, for values of $\Omega$ much larger than $\Delta$. For instance, for $\gamma_{\mathrm{fd}} / \Delta=0.001$ we observe differences for $\Omega / \Delta$ greater than 100. When this ratio overcomes a given value, we observe steady values of $r_{x}$ different from zero, with $r_{z}$ becoming positive but very close to zero, and at a certain point the nonsecular MME starts to predict nonphysical steady states. The occurrence of differences between secular and nonsecular master equations has been discussed in Ref. [44].

In order to show how different steady states for the same values of the control field parameters are dynamically obtained, we report in Fig. 3 the time evolution of $r_{x}$ and $r_{z}$ for the same cases of the points highlighted in Fig. 2(a), obtained with $\Omega=\sqrt{2} \Delta$ and $\varphi=\pi$. In particular, we choose $\gamma_{-}=0.2 \gamma_{0}$ and $\gamma_{+}=2 \gamma_{0}$ for the case $\mu=0.1$, the FDME for the fixed dissipator case $(\mu=1)$, and $\gamma_{-}=2 \gamma_{0}$ and $\gamma_{+}=$ $0.2 \gamma_{0}$ for the case $\mu=10$. The FDME dynamics is practically identical to the one of the MME in the limit of flat spectrum, $\gamma_{-}=\gamma_{+}=\gamma_{0}=\gamma_{\mathrm{fd}}(\mu=1)$ for values of $\gamma_{\mathrm{fd}}$ small enough, as in this case where $\gamma_{\mathrm{fd}}=0.001 \Delta$. The qubit is initially in the ground state $|g\rangle$. The values of the rates $\gamma_{p}$ are chosen without referring to a specific spectral density (no Lamb shift is considered).

We have then shown that, in general, using the FDME can cause a lack of accuracy in determining the steady state, which would be detrimental in a quantum control protocol. This effect can be enlightened by considering the distance between the stationary state induced by a structured spectral density, as predicted by the MME, and the one given by the FDME as a function of the control field parameter $\Omega / \Delta$ (this distance does not depend on $\varphi$ ). In Fig. 4, we use the fidelity 


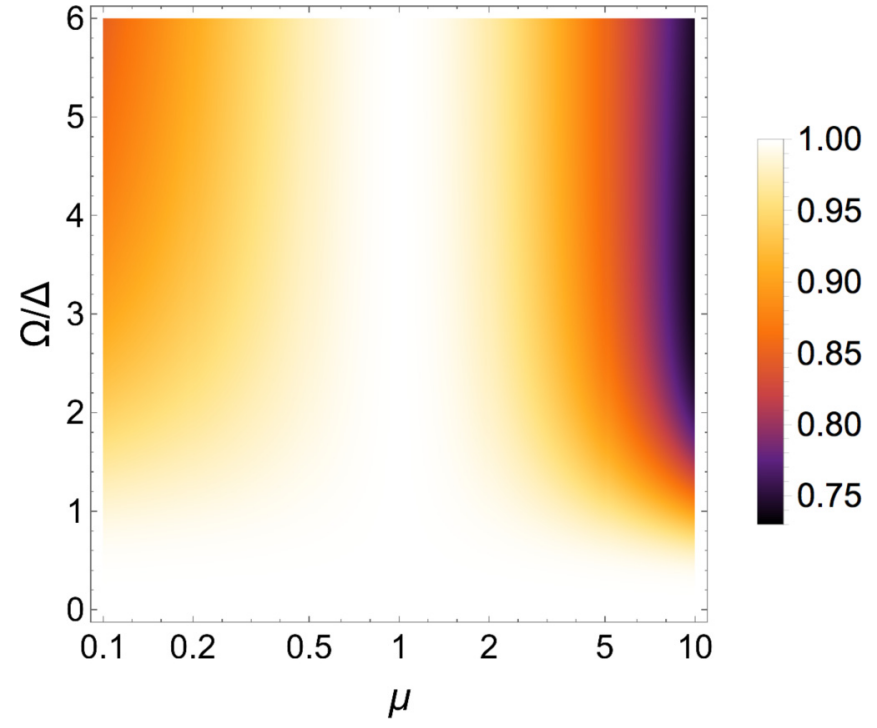

FIG. 4. Fidelity between the FDME steady states (their dependence on $\gamma_{\mathrm{fd}}$ is assumed to be negligible) and the ones obtained with the secular MME by releasing the flat-spectrum assumption, as a function of the control field parameter $\Omega / \Delta$ and of the ratio $\mu=\gamma_{-} / \gamma_{+}$(in logarithmic scale).

as a measure of such distance. For two arbitrary states $\rho$ and $\sigma$, it is defined as $[\operatorname{Tr}\{\sqrt{\sqrt{\rho} \sigma \sqrt{\rho}}\}]^{2}$ [49]. It is important to stress that a fidelity of the order of $3 / 4$ is already an indication of a dramatic difference between two states. For instance, the fidelity between a two-qubit Bell state and the state obtained from it by removing the coherence is $1 / \sqrt{2}$. In Fig. 4 , an important discrepancy may be observed for $\Omega / \Delta \gtrsim 1$. In particular, for a given value of $\Omega / \Delta$, smaller values of fidelity are obtained when $\mu$ moves away from 1 . The behavior for $\Omega / \Delta<1$ is instead reminiscent of the fact that for small angles $\theta$ the microscopic dissipator tends to the FD one, as shown before Eq. (16).

One may raise doubts about the freedom in the choice of the spectral density. In particular, the Markovian approximation could break down for some frequency region. Here, we remark that the results of this section hold for values of the system-bath coupling such that we are well behind the weakcoupling limit and the Markovian character of the dynamics is warranted. In any case, even for an intermediate coupling constant, we are interested in the stationary regime that takes place long after all the possible non-Markovian effects have been washed out.

\section{B. The case of nonzero temperature}

According to what has been said so far, a structured spectral density allows for a broader family of target states but, at the same time, would typically give solutions that are distinct from the ellipsoid predicted by the FDME. We now show that zero-temperature FDME steady states can be recovered in the case of a structured environment by exploiting tailored thermal effects.

To this aim, we consider the FDME steady states of Eq. (17) in the limit when the terms depending on $\gamma_{\mathrm{fd}}$ are

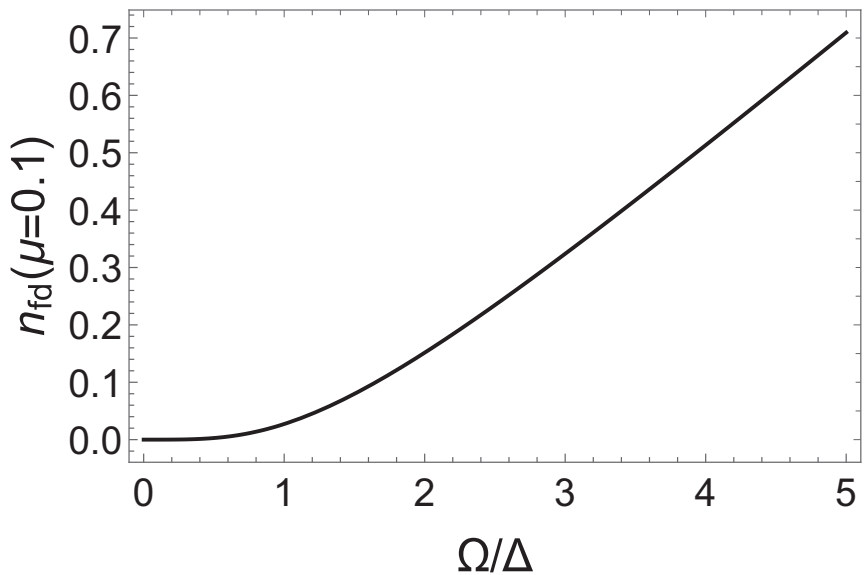

FIG. 5. Thermal factor $n_{\mathrm{fd}}(\mu=0.1)$ as a function of $\Omega / \Delta$ necessary to compensate the effects due to a ratio $\mu=\gamma_{-} / \gamma_{+}=0.1$ in order for the MME steady state at the temperature corresponding to $n_{\mathrm{fd}}(\mu=0.1)$ to end up in the zero-temperature flat-spectrum ellipsoid formed by the (approximated) FDME steady states.

negligible (being always $n_{-} \approx n_{+} \approx n_{0} \approx n_{\mathrm{fd}}$ ):

$$
\begin{aligned}
& \rho_{e e}^{\mathrm{sec}}(\mu=1) \approx \frac{n_{\mathrm{fd}}}{1+2 n_{\mathrm{fd}}}+\frac{\Omega^{2} /\left(1+2 n_{\mathrm{fd}}\right)}{4 \Delta^{2}+2 \Omega^{2}}, \\
& \rho_{e g}^{\mathrm{sec}}(\mu=1) \approx-\frac{\Omega \Delta /\left(1+2 n_{\mathrm{fd}}\right)}{2 \Delta^{2}+\Omega^{2}} e^{-i \varphi} .
\end{aligned}
$$

We indicate them with superscript "sec" since they coincide with the steady states of the secular MME [see Eq. (19)] in the limit of flat spectrum $(\mu=1)$. We compare them at zero temperature with the general case of Eq. (19) that depends both on $n_{\mathrm{fd}}$ and on the ratio $\mu=\gamma_{-} / \gamma_{+}$, and look, for any given $\mu$, for the existence of solutions of

$$
\begin{aligned}
& \rho_{e e}^{\mathrm{sec}}\left(\mu=1, n_{\mathrm{fd}}=0\right)=\rho_{e e}^{\mathrm{sec}}\left(\mu, n_{\mathrm{fd}}\right) \\
& \rho_{e g}^{\mathrm{sec}}\left(\mu=1, n_{\mathrm{fd}}=0\right)=\rho_{e g}^{\mathrm{sec}}\left(\mu, n_{\mathrm{fd}}\right)
\end{aligned}
$$

The solution of both equations is given by

$$
n_{\mathrm{fd}}(\mu)=\frac{S^{4} C^{4}(1-\mu)}{\left(C^{2}-S^{2}\right)\left(C^{4}+\mu S^{4}\right)} .
$$

Solutions corresponding to physical values of $n_{\mathrm{fd}}$ (that is $n_{\mathrm{fd}} \geqslant$ 0 ) only appear for $0 \leqslant \mu \leqslant 1$, which is easy to understand looking at Fig. 2(a). In fact, thermal effects are expected to reduce the value of $\left|\rho_{e g}\right|$ of any state, making it impossible to move from the black line $(\mu=10)$ to the blue one $(\mu=1)$. The behavior of $n_{\mathrm{fd}}(\mu=0.1)$ is plotted in Fig. 5 as a function of the control field parameter $\Omega / \Delta$. The needed thermal correction is very small as long as $\Omega<\Delta$, as the same argument used to explain the behavior observed in Fig. 4 holds.

\section{DISCUSSION AND CONCLUSIONS}

Master equations are a powerful tool to analyze the dissipative dynamics of quantum systems. They are usually obtained by making a series of assumptions that need to be fulfilled and to be verified in realistic setups, as, in general, exact solutions are not available. They are often introduced on the basis on phenomenological assumptions. Here, we have 
derived a microscopic master equation for a driven qubit and compared it with the fixed dissipator model, which is widely used, especially in the quantum control community, as it allows one to explore the behavior of entire families of control Hamiltonians in a simple way. We have found that, in the weak-coupling regime, the steady states of the two approaches can be very different in the case of a structured environment, while they are practically identical for a flat spectrum.

In conclusion, considering the simplest case of a driven qubit, we have assessed the limit of validity of the phenomenological approach for the specific task on asymptotic timescales. We have explored the possibility of implementing reservoir engineering techniques to widen the family of target states, which are correctly predicted by using microscopic master equations.

Quantum control protocols most often use time-dependent fields, implying time-dependent Rabi frequency, detuning, and phase as control parameters. For slowly varying parameters, one expects that the FDME and MME still coincide for a flat environment spectrum and that the difference between them still persists for structured environments. The expected rich variety of target states resulting from structured environments could then be exploited using microscopic models in quantum control and reservoir engineering schemes.

\section{ACKNOWLEDGMENTS}

This work was mainly supported by the French "Investissements d'Avenir" program, Project ISITE-BFC (Contract No. ANR-15-IDEX-03). G.L.G. acknowledges financial support from the María de Maeztu Program for Units of Excellence in R\&D (MDM-2017-0711) and the CAIB postdoctoral program. A.S. and S.G. acknowledge additional support from the European Union's Horizon 2020 research and innovation program under the Marie Sklodowska-Curie Grant Agreement No. 765075 (LIMQUET). G.L.G. and B.B. thank HansRudolf Jauslin, Axel Kuhn, and David Viennot for useful discussions. B.B. thanks Mauro Paternostro for helpful comments.

\section{APPENDIX: MASTER EQUATION}

In this Appendix, we derive the microscopic master equation of the driven qubit, presenting its various parts. Using Eq. (8), in the interaction picture with respect to $H_{S}+H_{E}$, the interaction Hamiltonian of Eq. (5) reads

$$
\tilde{H}_{I}(t)=B(t)\left(f_{+}^{t} \tilde{\sigma}_{+}+f_{-}^{t} \tilde{\sigma}_{-}+f_{z}^{t} \tilde{\sigma}_{z}\right),
$$

with $B(t)=\sum_{k} \hbar\left(g_{k} a_{k} e^{-i \omega_{k} t}+g_{k}^{*} a_{k}^{\dagger} e^{i \omega_{k} t}\right)$ and where

$$
\begin{aligned}
f_{+}^{t} & =\left[C^{2} e^{i\left(\omega_{L} t+\varphi\right)}-S^{2} e^{-i\left(\omega_{L} t+\varphi\right)}\right] e^{i v t}, \\
f_{-}^{t} & =\left[C^{2} e^{-i\left(\omega_{L} t+\varphi\right)}-S^{2} e^{i\left(\omega_{L} t+\varphi\right)}\right] e^{-i v t}, \\
f_{z}^{t} & =S C\left[e^{i\left(\omega_{L} t+\varphi\right)}+e^{-i\left(\omega_{L} t+\varphi\right)}\right] .
\end{aligned}
$$

The operators entering the master equation are multiplied by $f_{i}^{t} f_{j}^{t-s}$, with $i, j=+,-, z$. Thus, there will be secular terms for $\{i, j\}$ such that $f_{i}^{t}=f_{j}^{t *}$ and nonsecular terms in all other cases. In general, the products $f_{i}^{t} f_{j}^{t-s}$ may have parts oscillating at the laser frequency $e^{ \pm 2 i \omega_{L} t}$. For instance,

$$
\begin{aligned}
f_{+}^{t} f_{+}^{t-s}= & \left\{C^{4} e^{i\left[\omega_{L}(2 t-s)+2 \varphi\right]}+S^{4} e^{-i\left[\omega_{L}(2 t-s)+2 \varphi\right]}\right. \\
& \left.-2 S^{2} C^{2} \cos \omega_{L} s\right\} e^{i \nu(2 t-s)} .
\end{aligned}
$$

On the basis of the condition assumed in Sec. II, $\omega_{L} \gg \Delta, \Omega$, we note that the first two fast-oscillating terms in Eq. (A3) can be neglected. We observe that neglecting this kind of terms is completely equivalent to obtaining the master equation writing the interaction Hamiltonian in rotating wave approximation:

$$
H_{I}=\sum_{k} \hbar\left[g_{k} a_{k} e^{i\left(\omega_{L} t+\varphi\right)} \sigma_{+}+g_{k}^{*} a_{k}^{\dagger} e^{-i\left(\omega_{L} t+\varphi\right)} \sigma_{-}\right] .
$$

In this limit, the products linked to the secular terms

$$
\begin{aligned}
f_{-}^{t} f_{+}^{t-s} & \approx\left[C^{4} e^{-i \omega_{L} s}+S^{4} e^{i \omega_{L} s}\right] e^{-i \nu s}, \\
f_{+}^{t} f_{-}^{t-s} & \approx\left[C^{4} e^{i \omega_{L} s}+S^{4} e^{-i \omega_{L} s}\right] e^{i \nu s}, \\
f_{z}^{t} f_{z}^{t-s} & \approx 2 S^{2} C^{2} \cos \omega_{L} s
\end{aligned}
$$

determine the coefficients of Eq. (11). Nonsecular terms are determined by the products

$$
\begin{aligned}
& f_{+}^{t} f_{+}^{t-s} \approx-2 S^{2} C^{2} e^{i v(2 t-s)} \cos \omega_{L} s, \\
& f_{+}^{t} f_{z}^{t-s} \approx S C\left(C^{2} e^{i \omega_{L} s}-S^{2} e^{-i \omega_{L} s}\right) e^{i v t}, \\
& f_{z}^{t} f_{+}^{t-s} \approx S C\left(C^{2} e^{-i \omega_{L} s}-S^{2} e^{i \omega_{L} s}\right) e^{i \nu(t-s)},
\end{aligned}
$$

together with $f_{-}^{t} f_{-}^{t-s}=\left(f_{+}^{t} f_{+}^{t-s}\right)^{*}, f_{-}^{t} f_{z}^{t-s}=\left(f_{+}^{t} f_{z}^{t-s}\right)^{*}$, and $f_{z}^{t} f_{-}^{t-s}=\left(f_{z}^{t} f_{+}^{t-s}\right)^{*}$. The factors $e^{ \pm i v t}$ and $e^{ \pm 2 i v t}$ are taken out when one moves back to the Schrödinger picture. We indicate with $\overline{f_{i}^{t} f_{j}^{t-s}}$ the products $f_{i}^{t} f_{j}^{t-s}$ after the elimination of the factors $e^{ \pm i v t}$ and $e^{ \pm 2 i v t}$ and, taking the continuum limit, we introduce the spectral density $J(\omega)=\sum_{k}\left|g_{k}\right|^{2} \delta\left(\omega-\omega_{k}\right)$, such that the trace over the bath's degrees of freedom is transformed into an integral over all the frequencies. The Born-Markov master equation, assuming a factorized initial condition for the system and its bath, is then given by $[1,2]$

$$
\begin{aligned}
\dot{\rho}= & -\frac{i}{\hbar}\left[H_{S}, \rho\right]+\frac{1}{\hbar^{2}} \sum_{i, j=+,-, z} \int_{0}^{\infty} d s \\
& {\left[\overline{f_{i}^{t *} f_{j}^{t-s}}\langle B(t) B(t-s)\rangle\left(\tilde{\sigma}_{j} \rho \tilde{\sigma}_{i}^{\dagger}-\tilde{\sigma}_{i}^{\dagger} \tilde{\sigma}_{j} \rho\right)+\text { H.c. }\right], }
\end{aligned}
$$

where H.c. denotes Hermitian conjugation and the bath correlation functions, taking a thermal equilibrium state $\rho_{B}$ at temperature $T$, are given by

$$
\begin{aligned}
& \operatorname{Tr}_{B}\left\{B(t) B(t-s) \rho_{B}\right\} \\
& \quad=\hbar^{2} \int_{0}^{\infty} d \omega J(\omega)\left\{[1+n(\omega)] e^{-i \omega s}+n(\omega) e^{i \omega s}\right\} .
\end{aligned}
$$

The explicit development of Eq. (A7) leads to Eq. (9). In particular, in order to calculate the coefficients of the master equation, one makes use of the identity

$$
\int_{0}^{\infty} e^{ \pm i \varepsilon s} d s=\pi \delta(\varepsilon) \pm i \mathcal{P} \frac{1}{\varepsilon},
$$

where $\delta(\varepsilon)$ is the Dirac $\delta$ function and $\mathcal{P}$ denotes the Cauchy principal value. 
The Lamb shift Hamiltonian of Eq. (9) is given by

$$
H_{L S}=\hbar\left(s_{-}^{\theta} \tilde{\sigma}_{-} \tilde{\sigma}_{+}+s_{+}^{\theta} \tilde{\sigma}_{+} \tilde{\sigma}_{-}+s_{z}^{\theta} \tilde{\sigma}_{z}^{2}\right) \text {, }
$$

where

$$
\begin{aligned}
& s_{-}^{\theta}=\mathcal{P} \int_{0}^{\infty} d \omega J(\omega)\left\{\frac{S^{4}[1+n(\omega)]}{\left(\omega_{L}-v\right)-\omega}-\frac{C^{4} n(\omega)}{\left(\omega_{L}+v\right)-\omega}\right\}, \\
& s_{+}^{\theta}=\mathcal{P} \int_{0}^{\infty} d \omega J(\omega)\left\{\frac{C^{4}[1+n(\omega)]}{\left(\omega_{L}+v\right)-\omega}-\frac{S^{4} n(\omega)}{\left(\omega_{L}-v\right)-\omega}\right\}, \\
& s_{z}^{\theta}=\mathcal{P} \int_{0}^{\infty} d \omega J(\omega) \frac{S^{2} C^{2}}{\omega_{L}-\omega} .
\end{aligned}
$$

We observe that it holds $\left[H_{S}, H_{L S}\right]=0$.

As for the nonsecular part $\mathcal{D}^{\text {nsec }}(\rho)$, we have

$$
\begin{aligned}
\mathcal{D}^{\mathrm{nsec}}(\rho)= & \left(\gamma_{++}^{\theta}+i s_{++}^{\theta}\right) \tilde{\sigma}_{+} \rho \tilde{\sigma}_{+} \\
& +\left(\gamma_{+z}^{\theta}+i s_{+z}^{\theta}\right)\left(\tilde{\sigma}_{+} \tilde{\sigma}_{z} \rho-\tilde{\sigma}_{z} \rho \tilde{\sigma}_{+}\right) \\
& +\left(\gamma_{-z}^{\theta}+i s_{-z}^{\theta}\right)\left(\tilde{\sigma}_{-} \tilde{\sigma}_{z} \rho-\tilde{\sigma}_{z} \rho \tilde{\sigma}_{-}\right) \\
& +\left(\gamma_{z+}^{\theta}+i s_{z+}^{\theta}\right)\left(\tilde{\sigma}_{z} \tilde{\sigma}_{+} \rho-\tilde{\sigma}_{+} \rho \tilde{\sigma}_{z}\right) \\
& +\left(\gamma_{z-}^{\theta}+i s_{z-}^{\theta}\right)\left(\tilde{\sigma}_{z} \tilde{\sigma}_{-} \rho-\tilde{\sigma}_{-} \rho \tilde{\sigma}_{z}\right)+\text { H.c. }
\end{aligned}
$$

where the various coefficients $\gamma_{i j}^{\theta}$ and $s_{i j}^{\theta}$ can be computed by explicitly developing Eq. (A7):

$$
\begin{aligned}
\gamma_{++}^{\theta} & =-\frac{1}{2} S^{2} C^{2}\left[\gamma_{-}\left(1+2 n_{-}\right)+\gamma_{+}\left(1+2 n_{+}\right)\right], \\
\gamma_{z+}^{\theta} & =-\frac{1}{2} S C\left[\gamma_{+} n_{+} C^{2}-\gamma_{-}\left(1+n_{-}\right) S^{2}\right], \\
\gamma_{z-}^{\theta} & =-\frac{1}{2} S C\left[\gamma_{+}\left(1+n_{+}\right) C^{2}-\gamma_{-} n_{-} S^{2}\right], \\
\gamma_{+z}^{\theta} & =-\frac{1}{2} \gamma_{0} S C\left[\left(1+n_{0}\right) C^{2}-n_{0} S^{2}\right], \\
\gamma_{-z}^{\theta} & =-\frac{1}{2} \gamma_{0} S C\left[n_{0} C^{2}-\left(1+n_{0}\right) S^{2}\right],
\end{aligned}
$$

and

$$
\begin{aligned}
s_{++}^{\theta}= & -\mathcal{P} \int_{0}^{\infty} d \omega J(\omega) S^{2} C^{2} \\
& \times\left[\frac{1+2 n(\omega)}{\left(\omega_{L}-v\right)-\omega}-\frac{1+2 n(\omega)}{\left(\omega_{L}+v\right)-\omega}\right],
\end{aligned}
$$

$$
\begin{aligned}
s_{z+}^{\theta}= & \mathcal{P} \int_{0}^{\infty} d \omega J(\omega) S C \\
& \times\left\{\frac{S^{2}[1+n(\omega)]}{\left(\omega_{L}-v\right)-\omega}+\frac{C^{2} n(\omega)}{\left(\omega_{L}+v\right)-\omega}\right\}, \\
s_{z-}^{\theta}= & -\mathcal{P} \int_{0}^{\infty} d \omega J(\omega) S C \\
& \times\left\{\frac{S^{2} n(\omega)}{\left(\omega_{L}-v\right)-\omega}+\frac{C^{2}[1+n(\omega)]}{\left(\omega_{L}+v\right)-\omega}\right\}, \\
s_{+z}^{\theta}= & -\mathcal{P} \int_{0}^{\infty} d \omega J(\omega) S C\left[\frac{C^{2}+n(\omega)}{\omega_{L}-\omega}\right], \\
s_{-z}^{\theta}= & \mathcal{P} \int_{0}^{\infty} d \omega J(\omega) S C\left[\frac{S^{2}+n(\omega)}{\omega_{L}-\omega}\right] .
\end{aligned}
$$

For each pair $\{i, j\}$ in Eq. (A7), the part of the integrals involving the $\delta$ function gives us the decay rates of Eq. (11) when $i=j$ and the ones of Eq. (A13) when $i \neq j$, for any spectral density. The principal part in Eq. (A9) leads to the Lamb shift Hamiltonian of Eq. (A10) and the terms in Eq. (A14). Note that the subscripts of $\gamma_{i}^{\theta}, s_{i}^{\theta}, \gamma_{i j}^{\theta}$, and $s_{i j}^{\theta}$ are chosen independently of the actual values of $i$ and $j$ in Eq. (A7), leading to the various terms where these parameters appear.

It can be shown (see, for instance, Ref. [41]) that, in the case of a flat spectral density, all the terms deriving from the principal part of Eq. (A9) vanish. This can be obtained by first performing the integrals by using a Lorentzian spectral density and by then taking the width of this Lorentzian to infinity. In the case of a nonflat spectrum, we treat these terms, taking again the Lorentzian spectral density. In the secular MME, the terms in the Lamb shift Hamiltonian lead to nothing but energy shift, and then their effect is not relevant for the steady states. Instead, the contribution of the terms deriving from the principal part of Eq. (A9) appearing in the nonsecular MME [see Eq. (A14)], in general, cannot be neglected (see comment in Sec. V A on the comparison between secular and nonsecular master equations).

Finally, keeping the terms of Eq. (A12) in Eq. (9), it is possible to show that in the flat-spectrum limit, under the approximation $n_{-} \approx n_{+} \approx n_{0} \approx n_{\mathrm{fd}}$, the nonsecular MME gives exactly the same result as the FDME; i.e., using $H=H_{S}$, Eq. (9) becomes Eq. (13) for any $\gamma_{\mathrm{fd}}$.
[1] H.-P. Breuer and F. Petruccione, The Theory of Open Quantum Systems (Oxford University Press, New York, 2007).

[2] C. W. Gardiner and P. Zoller, Quantum Noise (Springer-Verlag, Berlin, 2000).

[3] D. D'Alessandro, Introduction to Quantum Control and Dynamics (Chapman \& Hall/CRC, Boca Raton, FL, 2007).

[4] A. Carlini, A. Hosoya, T. Koike, and Y. Okudaira, Phys. Rev. Lett. 96, 060503 (2006).

[5] D. Sugny, C. Kontz, and H. R. Jauslin, Phys. Rev. A 76, 023419 (2007).

[6] M. Lapert, Y. Zhang, M. Braun, S. J. Glaser, and D. Sugny, Phys. Rev. Lett. 104, 083001 (2010).

[7] M. Shapiro and P. Brumer, Quantum Control of Molecular Processes, 2nd ed. (Wiley Interscience, New York, 2012).
[8] S. J. Glaser, U. Boscain, T. Calarco, C. P. Koch, W. Köckenberger, R. Kosloff, I. Kuprov, B. Luy, S. Schirmer, T. Schulte-Herbrüggen, D. Sugny, and F. K. Wilhelm, Eur. Phys. J. D 69, 279 (2015).

[9] C. P. Koch, J. Phys.: Condens. Matter 28, 213001 (2016).

[10] C. H. Bennett, D. P. DiVincenzo, P. W. Shor, J. A. Smolin, B. M. Terhal, and W. K. Wootters, Phys. Rev. Lett. 87, 077902 (2001)

[11] N. A. Peters, J. T. Barreiro, M. E. Goggin, T.-C. Wei, and P. G. Kwiat, Phys. Rev. Lett. 94, 150502 (2005).

[12] L. Viola and S. Lloyd, Phys. Rev. A 58, 2733 (1998).

[13] L. Viola, E. Knill, and S. Lloyd, Phys. Rev. Lett. 82, 2417 (1999). 
[14] C. Addis, F. Ciccarello, M. Cascio, G. M. Palma, and S. Maniscalco, New J. Phys. 17, 123004 (2015).

[15] L. Hartmann, W. Dür, and H.-J. Briegel, Phys. Rev. A 74, 052304 (2006).

[16] H. Krauter, C. A. Muschik, K. Jensen, W. Wasilewski, J. M. Petersen, J. I. Cirac, and E. S. Polzik, Phys. Rev. Lett. 107, 080503 (2011).

[17] B. Bellomo, R. Messina, D. Felbacq, and M. Antezza, Phys. Rev. A 87, 012101 (2013).

[18] B. Bellomo and M. Antezza, Phys. Rev. A 91, 042124 (2015).

[19] G. Lindblad, Commun. Math. Phys. 48, 119 (1976).

[20] V. Gorini, A. Kossakowski, and E. C. G. Sudarshan, J. Math. Phys. 17, 821 (1976).

[21] J. O. González, L. A. Correa, G. Nocerino, J. P. Palao, D. Alonso, and G. Adesso, Open Syst. Inf. Dyn. 24, 1740010 (2017).

[22] M. T. Naseem, A. Xuereb, and Ö. E. Müstecaplığlu, Phys. Rev. A 98, 052123 (2018).

[23] M. Cattaneo, G. L. Giorgi, S. Maniscalco, and R. Zambrini, New J. Phys. 21, 113045 (2019).

[24] X. Lacour, S. Guérin, and H. R. Jauslin, Phys. Rev. A 78, 033417 (2008).

[25] S. Sauer, C. Gneiting, and A. Buchleitner, Phys. Rev. Lett. 111, 030405 (2013).

[26] M. Lapert, E. Assémat, S. J. Glaser, and D. Sugny, Phys. Rev. A 88, 033407 (2013).

[27] V. Mukherjee, A. Carlini, A. Mari, T. Caneva, S. Montangero, T. Calarco, R. Fazio, and V. Giovannetti, Phys. Rev. A 88, 062326 (2013).

[28] S. Sauer, C. Gneiting, and A. Buchleitner, Phys. Rev. A 89, 022327 (2014).

[29] M. Scala, B. Militello, A. Messina, J. Piilo, and S. Maniscalco, Phys. Rev. A 75, 013811 (2007).

[30] Á. Rivas, A. Douglas, K. Plato, S. F. Huelga, and M. B. Plenio, New J. Phys. 12, 113032 (2010).
[31] F. Beaudoin, J. M. Gambetta, and A. Blais, Phys. Rev. A 84, 043832 (2011).

[32] T. Werlang, M. A. Marchiori, M. F. Cornelio, and D. Valente, Phys. Rev. E 89, 062109 (2014).

[33] J. Kołodyński, J. B. Brask, M. Perarnau-Llobet, and B. Bylicka, Phys. Rev. A 97, 062124 (2018).

[34] M. Scala, B. Militello, A. Messina, and N. V. Vitanov, Phys. Rev. A 81, 053847 (2010).

[35] B. Militello, M. Scala, A. Messina, and N. V. Vitanov, Phys. Scr. T143, 014019 (2011).

[36] M. Scala, B. Militello, A. Messina, and N. V. Vitanov, Opt. Spectrosc. 111, 589 (2011).

[37] A. Levy and R. Kosloff, Europhys. Lett. 107, 20004 (2014).

[38] G. De Chiara, G. Landi, A. Hewgill, A. Ferraro, A. J. Roncaglia, and M. Antezza, New J. Phys. 20, 113024 (2018).

[39] C. Addis, E.-M. Laine, C. Gneiting, and S. Maniscalco, Phys. Rev. A 94, 052117 (2016).

[40] G. Shavit, B. Horovitz, and M. Goldstein, Phys. Rev. B 100, 195436 (2019).

[41] A. Kowalewska-Kudlaszyk and R. Tanaś, J. Mod. Opt. 48, 347 (2001).

[42] P. Haikka and S. Maniscalco, Phys. Rev. A 81, 052103 (2010).

[43] U. Weiss, Quantum Dissipative Systems, 4th ed. (World Scientific, Singapore, 2012).

[44] H. Z. Shen, M. Qin, X.-M. Xiu, and X. X. Yi, Phys. Rev. A 89, 062113 (2014).

[45] B. Recht, Y. Maguire, S. Lloyd, I. L. Chuang, and N. A. Gershenfeld, arXiv:quant-ph/0210078.

[46] M. H. Levitt, Spin Dynamics: Basics of Nuclear Magnetic Resonance (Wiley, New York, 2008).

[47] R. R. Ernst, G. Bodenhausen, and A. Wokaun, Principles of Nuclear Magnetic Resonance in One and Two Dimensions (Clarendon Press, Oxford, UK, 1987), Vol. 14.

[48] A. Streltsov, G. Adesso, and M. B. Plenio, Rev. Mod. Phys. 89, 041003 (2017).

[49] R. Jozsa, J. Mod. Opt. 41, 2315 (1994). 